\title{
A Descriptive Comparison of Alcohol-Related Presentations at a Large Urban Hospital Center from 1902 to 2009
}

\author{
Bradley D. Shy $\cdot$ Robert S. Hoffman
}

Published online: 4 May 2012

(C) American College of Medical Toxicology 2012

\begin{abstract}
Although alcohol use has long been a significant cause of hospital presentations, little is published regarding the long-term demographic changes that have occurred at a single hospital site. To address this deficit, we prospectively studied all acute alcohol-related presentations to Bellevue Hospital Center (New York, NY) and compared this contemporary data set with one from the same institution from 1902 to 1935 . We prospectively identified all patients presenting to the emergency department because of acute alcohol use over an 8-week period in 2009. We described the basic attributes of patients presenting currently because of alcohol and compared these data to those previously described between 1902 and 1935. We also compared our census data with contemporaneous data from all patients presenting to this hospital site. During the study period, 560 patients presented because of acute alcohol use which extrapolated to an estimated 3,800 patients over the calendar year. This compares to 7,600 presentations recorded annually early in the twentieth century. Twelve percent of patients in 2009 were female as compared to $18 \%$ of patients between 1934 and 1935. Patients with alcohol-related presentations in
\end{abstract}

\footnotetext{
B. D. Shy $(\bowtie)$

Department of Emergency Medicine,

Mount Sinai School of Medicine,

One Gustave L. Levy Place-Box 1620,

New York, NY 10029-6574, USA

e-mail: brad.shy@gmail.com

R. S. Hoffman

Department of Emergency Medicine,

New York University School of Medicine,

New York, NY, USA
}

2009 were more likely to be admitted than contemporaneous patients without an alcohol-related presentation (30 vs. $19 \%$ admitted; $p<0.001)$. Since first measured 110 years ago at one large New York City hospital, alcohol-related presentations remain common representing $5 \%$ of all emergency department visits. This demonstrates alcoholism's continuing toll on society's limited medical resources and on public health as a whole.

Keywords Alcohol · Demographics · Emergency department $\cdot$ Emergency medicine $\cdot$ Urban

\section{Introduction}

Alcohol use has been a significant cause of hospital visits for centuries [1]. These presentations are not limited to simple alcohol intoxication, but rather encompass addiction, withdrawal, and trauma and neurological, nutritional, hepatic, gastrointestinal, and psychiatric disease [2-5]. Numerous studies have described the impact of alcohol on hospital and emergency department (ED) censuses, although these have typically focused on a single point in time [5-7].

One of the longest-running single hospital surveys of alcohol-related presentations was conducted at Bellevue Hospital Center over the first half of the twentieth century. Published by neurologist Norman Jolliffe in Science in 1936 [8], the research group documented 256,755 patient presentations to Bellevue between 1902 and 1935. Jolliffe showed how prohibition and its 1933 repeal, as well as the preceding temperance movement, correlated to each year's total number of 
per capita alcohol presentations. Demographic and clinical details about these presentations were limited to patient gender and age, but the sheer volume of presentations recorded makes this study an important resource in understanding the role of alcohol in public health over the study time period.

To appreciate how the nature of alcohol-related presentations has changed at a single urban hospital site over the previous century, we prospectively conducted a census of all alcohol-related presentations to Bellevue Hospital Center and compared this 2009 data set with the previous data published by Jolliffe. A secondary objective of this census was to describe in detail key demographic features of this current patient population and compare these to the general hospital population over this same period.

\section{Materials and Methods}

\section{Study Design}

Using 24-h-a-day trained research associates dedicated to this census, we prospectively identified all patients presenting to the ED because of acute alcohol use or withdrawal, as defined by their treating physician. Data for this survey were collected over a single 8-week period in 2009.

Setting and Population

The study was conducted entirely through the Bellevue Hospital Center Emergency Department in New York, NY. Patients in the adult ED, pediatric/young adult ED, fast track/urgent care center, and ED intensive care unit (ICU) were eligible. No effort was made to survey patients in the outpatient clinic system, psychiatric ED, or those transferred from other hospitals directly to an inpatient service.

All nonpregnant patients, 18 years or older, were screened by research associates. Patients were included if their treating ED physician believed the patient "would have been unlikely to present to the ED without the acute use or withdrawal of alcohol." The research associates used a 22-item structured survey instrument (Appendix 1) obtaining objective information, such as vital signs, from the medical chart and interviewing the treating emergency physician to obtain the remaining data. The research associates collected information from either attending emergency physicians or directly from emergency medicine residents if these house staff had already discussed the case with the attending and the patient had already been physically evaluated by this attending physician. To ensure patient privacy and census completeness, no confidential patient identifiers were recorded. The institutional review board approved this study as well as an exemption to either inform or consent patients about their inclusion in the study; this allowed for a more comprehensive survey as we could include intoxicated and unconscious patients who are normally unable to give consent.

\section{Data Analysis}

Trained research associates transferred all census surveys into an electronic database. A representative sample of the first week's data was checked by the investigators for accuracy and was found to be without discrepancy.

We used International Statistical Classification of Diseases (ICD9) diagnostic coding to control for potential seasonal variability in our data, as our survey was conducted entirely during the summer months. Four numbers from the ICD9 data were recorded: the total number of patients presenting to Bellevue for any reason over an entire current calendar year (2008); the total number of patients presenting to Bellevue in 2008 with an ICD9 diagnosis related to alcohol use or disease; the number of patients presenting for any reason over the study period (June 8-August 2); and the number of patients presenting over the study period (June 8August 2) with an ICD9 diagnosis related to alcohol use or disease. Since the total ED census was slightly higher in the summer months $(1.1 \%$ more total ED patients per day during these summer dates) and patients with alcohol-related presentations were a slightly smaller fraction of total presentations in the summer months $(5.2 \%$ fewer of the summer patients had alcohol-related ICD9 codes), we used these data to extrapolate a seasonally adjusted estimate of alcoholrelated presentations over the calendar year.

Statistical comparisons were made using Pearson's uncorrected chi-square test, the Student's $t$ test, and $F$ tests. Because of the large size of the study groups, all comparisons were found to be significant $(p<0.05)$ using both $t$ tests and $F$ tests. As comparisons between the alcohol-related patient presentations and general ED presentations were not part of the primary hypothesis, we used a Bonferroni correction for these analyses and deemed any comparison with a $p$ value of less than $0.001(0.05 \times 20$ comparisons $)$ to be significant. We 
Table 1 Comparison of acute alcohol-related presentations to the general ED census

\begin{tabular}{|c|c|c|}
\hline & $\begin{array}{l}\text { Presenting due to } \\
\text { alcohol use }(n=560)\end{array}$ & $\begin{array}{l}\text { All patients, } 2008 \\
(n=78,142)\end{array}$ \\
\hline \multicolumn{3}{|l|}{ Age } \\
\hline $18-30$ & $23 \%(125)$ & $25 \%(19,656)$ \\
\hline $31-40$ & $23 \%(122)$ & $20 \%(15,493)$ \\
\hline $41-50$ & $24 \%(127)$ & $23 \%(17,586)$ \\
\hline $51-60$ & $20 \%(108)$ & $17 \%(13,571)$ \\
\hline $61-70$ & $8.6 \%(46)$ & $8.9 \%(6,962)$ \\
\hline $71+$ & $1.7 \%(9)$ & $6.2 \%(4,874)$ \\
\hline Unspecified age $^{a}$ & $(23)$ & \\
\hline \multicolumn{3}{|l|}{ Gender } \\
\hline Female & $12 \%(64)$ & $60 \%(47,239)$ \\
\hline Male & $88 \%(461)$ & $40 \%(30,903)$ \\
\hline Unspecified gender $^{\mathrm{a}}$ & $(53)$ & \\
\hline \multicolumn{3}{|l|}{ Ethnicity } \\
\hline Asian & $6.5 \%(26)$ & $9.2 \%(6,759)$ \\
\hline Black & $31 \%(125)$ & $27 \%(20,231)$ \\
\hline Hispanic & $23 \%(93)$ & $41 \%(30,297)$ \\
\hline White & $39 \%(155)$ & $22 \%(16,498)$ \\
\hline Unspecified ethnicity $^{\mathrm{a}}$ & $(161)$ & $(4,357)$ \\
\hline \multicolumn{3}{|l|}{ Expected disposition } \\
\hline Home & $70 \%(289)$ & $81 \%(63,569)$ \\
\hline Admission & $30 \%(125)$ & $19 \%(14,573)$ \\
\hline $\begin{array}{l}\text { Withdrawal } \\
\text { management }\end{array}$ & $16 \%(65)$ & $1.9 \%(1,538)$ \\
\hline Medical (all beds) & $6.5 \%(27)$ & $9.4 \%(7,315)$ \\
\hline Medical ICU & $3.6 \%(15)$ & $0.3 \%(270)$ \\
\hline Psychiatric & $3.6 \%(15)$ & $0.1 \%(87)$ \\
\hline Neurology & $0.5 \%(2)$ & $0.5 \%(372)$ \\
\hline Unspecified service $^{\mathrm{a}}$ & $1.9 \%(8)$ & $0.2 \%(150)$ \\
\hline Unspecified disposition $^{a}$ & $(146)$ & \\
\hline
\end{tabular}

Because of the large size of the study groups, all comparisons were found to be significant using both $t$ tests and $F$ tests

${ }^{a}$ Patients with unspecified data were excluded from percentage calculations

conducted our analysis using MedCalc 11.6, produced by MedCalc Software (Mariakerke, Belgium).

\section{Results}

In total, 560 patients were included in this 8-week survey. This number extrapolates to a seasonally corrected estimate of 3,800 patients over the calendar year 2009. Table 1
Table 2 Detailed characteristics of all patients presenting because of alcohol use $(N=560)$

Mode of arrival

Self/walk-in

$27 \%(115)$

Ambulance

$68 \%(293)$

Law enforcement custody

$5.6 \%(24)$

Unspecified $^{\mathrm{a}}$

Subjective presenting state

Intoxicated, verbal

$75 \%(316)$

Intoxicated, nonverbal $^{\mathrm{b}}$

$12 \%(52)$

Withdrawal

$6.1 \%(26)$

Normal (not intoxicated)

$7.1 \%(30)$

Unspecified $^{\mathrm{a}}$

(136)

Trauma

Not present

$82 \%(377)$

Present

$18 \%(84)$

Assault

$4.1 \%(19)$

Motor vehicle collision

$0.2 \%(1)$

Pedestrian struck

$0.9 \%(4)$

Fall

$9.1 \%(42)$

Unspecified trauma

$3.9 \%(18)$

Unspecified $^{\mathrm{a}}$

(99)

Residence

Private residence

$46 \%(121)$

Group home or nursing facility

Homeless (undomiciled)

$2.6 \%(7)$

$52 \%(137)$

Slept last night

Street

$11 \%(29)$

Shelter

$5.7 \%(15)$

Home of friend/family

$0.8 \%(2)$

Homeless, last night's sleeping

$34 \%(91)$

site unspecified

Other/unspecified $^{\text {a }}$

Known seizure history

No

$80 \%(231)$

Yes, on anti-epileptics

$6.6 \%(19)$

$13 \%(39)$

$1.7 \%(5)$

(289)

Unspecified/unknown ${ }^{\mathrm{a}}$

$5.8 \%(32)$

Depression

$2.4 \%(13)$

Schizophrenia/schizoaffective disorder

$2.2 \%(15)$

Bipolar disorder

$2.8 \%(21)$

$1 \%$ (6)

Recent suicide attempt/ideation

$87 \%(479)$

No known diagnosis

$87 \%(479)$

Cigarette use

$76 \%(105)$ 
Table 2 (continued)

\begin{tabular}{ll}
\hline Former user & $7.9 \%(11)$ \\
Denies use & $17 \%(23)$ \\
Unspecified/unknown $^{\mathrm{a}}$ & $(421)$ \\
Head CT anticipated $^{\mathrm{c}}$ & \\
Yes & $19 \%(72)$ \\
No & $81 \%(307)$ \\
Unspecified $^{\mathrm{a}}$ & $(181)$ \\
\hline
\end{tabular}

${ }^{a}$ Patients with unspecified data were excluded from percentage calculations

${ }^{\mathrm{b}}$ Nonverbal intoxication refers to inebriated patients lacking a sufficient level of consciousness to speak

c "Head CT anticipated" connotes that the treating physician intended to obtain a computerized tomography study of the patient's head, although we did not follow this to ensure that the CT order was actually executed

compares the basic demographic data of patients presenting because of alcohol during the study period with these demographics from the larger ED population. Patients presenting because of alcohol use were significantly more likely to be both male and white than the general ED population. They were more likely to be admitted (30 vs. $19 \%$; $p<$ 0.001 ), and those admissions were more likely to be to ICU beds than the general ED patient population as a whole (12 vs. $1.9 \% ; p<0.001)$. A detailed demographic description of the patients presenting due to alcohol use is presented in Table 2.

This estimated 3,800 alcohol-related patient visits to Bellevue Hospital Center in 2009 compares to an annual average of 7,600 such patients between 1902 and 1935 (which ranged from a peak of 11,307 cases in 1910 to a low of 2,091 cases in 1920). Five percent (27 patients) from the current 2009 sample was older than 65 , comparable to the $5 \%$ of patients presenting to Bellevue in the earlier 1902-1935 study who were older than 65 . Eighty-eight percent of alcohol-related patients from the 2009 sample were men, while $82 \%$ of presenting patients were men in the final 2 years of the previous study between 1934 and 1935.

\section{Discussion}

Our data showed that $18 \%$ of patients who presented because of alcohol had concomitant trauma. Methodological differences preclude direct comparisons to previous studies of alcohol use and trauma; however, data from this current study dovetail previous literature investigating the proportion of trauma patients who had used alcohol. A multi-site 1989 study of ED patients with trauma, for example, showed $9 \%$ to have detectable alcohol levels on breathalyzer testing [9]. A similar ED-based survey of urban trauma patients in 1991 demonstrated that $21 \%$ of presenting trauma patients had evidence of acute alcohol use in serum testing [2]. A 1993 study measuring self-reported alcohol use among patients suffering trauma in the county/public ED setting vs. a private, managed care ED recorded 19 and $8 \%$ alcohol use, respectively [4].

Previous studies of alcohol abuse among medical and surgical inpatients have shown remarkably high frequencies of alcohol dependence in these populations ranging from 7 to $25 \%$ [10-13]. A unique strength of this current study is that it focuses not on chronic usage patterns, but rather how commonly alcohol is the acute cause for hospital presentations. Although we did not study chronic alcohol usage patterns in our current study, we believe it is likely that a substantially higher fraction of ED patients in this hospital setting suffered from alcohol dependence but had ED presentations that were unrelated to alcohol use.

Notably, $52 \%$ of patients presenting because of alcohol were undomiciled in this current study. This is substantially more than the $20 \%$ of the general ED population at this same hospital who were undomiciled [5]. Possibly contributing to both these figures is the one block proximity between the Bellevue ED and a 600-bed homeless shelter. Previous studies have estimated the frequency of alcoholism in undomiciled populations between 18 and $44 \%[14,15]$. Similarly, a 1992 New York State survey showed that although undomiciled individuals were more likely to abstain completely from alcohol than the general population ( 39.8 vs. $26.4 \%$ ), the homeless were also more likely to be daily heavy drinkers (33.3 vs. $14.1 \%$ ) [16].

This current analysis is unique in that it focuses on the change of alcohol-related presentation at a single site over 107 years. The strength of comparison, however, also limits the applicability of these data to other sites. Bellevue Medical Center is unique because of its location in the urban center of New York City, large number of undomiciled patients, and ability to offer care to many patients without the ability to pay. These differences are likely reflected in the demographics of alcohol-related presentations to Bellevue. For instance, a similar urban hospital in Pachuca, Mexico reported $9.4 \%$ of their 
ED patients to have used alcohol within $6 \mathrm{~h}$ of presentation, compared with an estimated $4.9 \%$ of Bellevue patients who presented because of alcohol [17]. A recent survey of Tennessee urban EDs found a similar $4.9 \%$ of presenting patients to be acutely intoxicated with alcohol based on serum analysis [7]. A larger analysis data from the National Hospital Ambulatory Medical Care Survey estimated that $2.7 \%$ of ED visits were related to alcohol abuse [18]. In sum, depending on the method of data collection and the area of study, the frequency of contemporary alcohol-related ED visits is widely variable.

\section{Limitations}

We used a prospective, 24-h per day study design to ensure as high a degree of accuracy as possible. Still, it is likely that at least some patients were missed in the collection of these data. We believe that using the assessment of the treating emergency physician was the most accurate determination of the association between patients' presentations and the acute use or withdrawal from alcohol. Nevertheless, this strategy may have missed a small number patients that would have been included if our study had relied on inclusion data such as ethanol concentrations and chief complaints. We did not attempt to validate the accuracy of our prospectively collected census with retrospective data.

Our comparison of the study population from $2009(n=$ 560 ) with the ICD9-derived population from the same calendar time in $2008(n=69)$ demonstrates that ICD9 diagnoses would not have been a reliable means of capturing this patient population in our institution. A small number of patients had incomplete data sheets, due primarily to uncertainty about the data accuracy from the treating ED physician. Furthermore, our study was confined to the ED. No effort was made to include patients admitted directly to the hospital, although this practice is rare in the study hospital and we do not believe that their inclusion would have affected our data significantly. Any psychiatric patient who may have had an acute medical problem would have been evaluated first in the medical ED and thus would have been eligible for our study. As our research associates would be VHunable to physically conduct the survey in the psychiatric ED, we did not include patients treated solely in the psychiatric ED and cannot estimate what fraction of these patient presentations were secondary to alcohol use or withdrawal.

We collected data for only 8 weeks. We used ICD9-coding to estimate seasonal variance of both total ED population and the relative frequency of alcohol-related presentations. Both of these corrections were less than $6 \%$, so they only had minimal effects on our final estimate. Still, if there was a gross seasonal discrepancy in ICD9 accuracy (for example, patients with alcohol intoxication more likely to be given an accurate ICD9 diagnosis in the winter than in the summer), this would affect our estimate.

We did not address contextual changes to Bellevue Hospital Center and New York City over the previous century. Isolated gentrification to the surrounding neighborhood and increased accessibility of nearby hospitals may have altered the number of presentations to Bellevue Hospital without any absolute change in the prevalence of alcohol-related disease in New York City. Although our data show a moderate decline in alcohol cases, our data collection methods differed inherently from those used in the early 1936 study. Importantly, the 1936 paper by Jolliffe studied patients presenting to the psychiatric section of Bellevue Hospital, whereas our current study included those patients presenting through the medical ED. Thus, this relative decline is only an estimate of the true change in alcohol presentation frequency and one cannot make reliable deductions from this current data on how alcohol presentations have changed at this hospital site over the preceding 108 years.

\section{Conclusions}

At one large New York City public hospital ED, presentations related to acute alcohol use and withdrawal have remained common since first studied 110 years ago. From the current data set, $4.9 \%$ of presentations were related to acute alcohol use, representing a substantial proportion of hospital visits. Compared to improvements in areas of public health such as infectious disease and occupational injury, alcohol-related disease remains a major public health struggle [19, 20].

Acknowledgments The authors thank Ashley Colucci, Ian Portrelli, Nick Williams, and the 32 other project healthcare volunteers; without their around-the-clock diligent work, this study would not have been possible. 


\section{Appendix 1}

\section{Alcohol Census Data Collection Instrument}

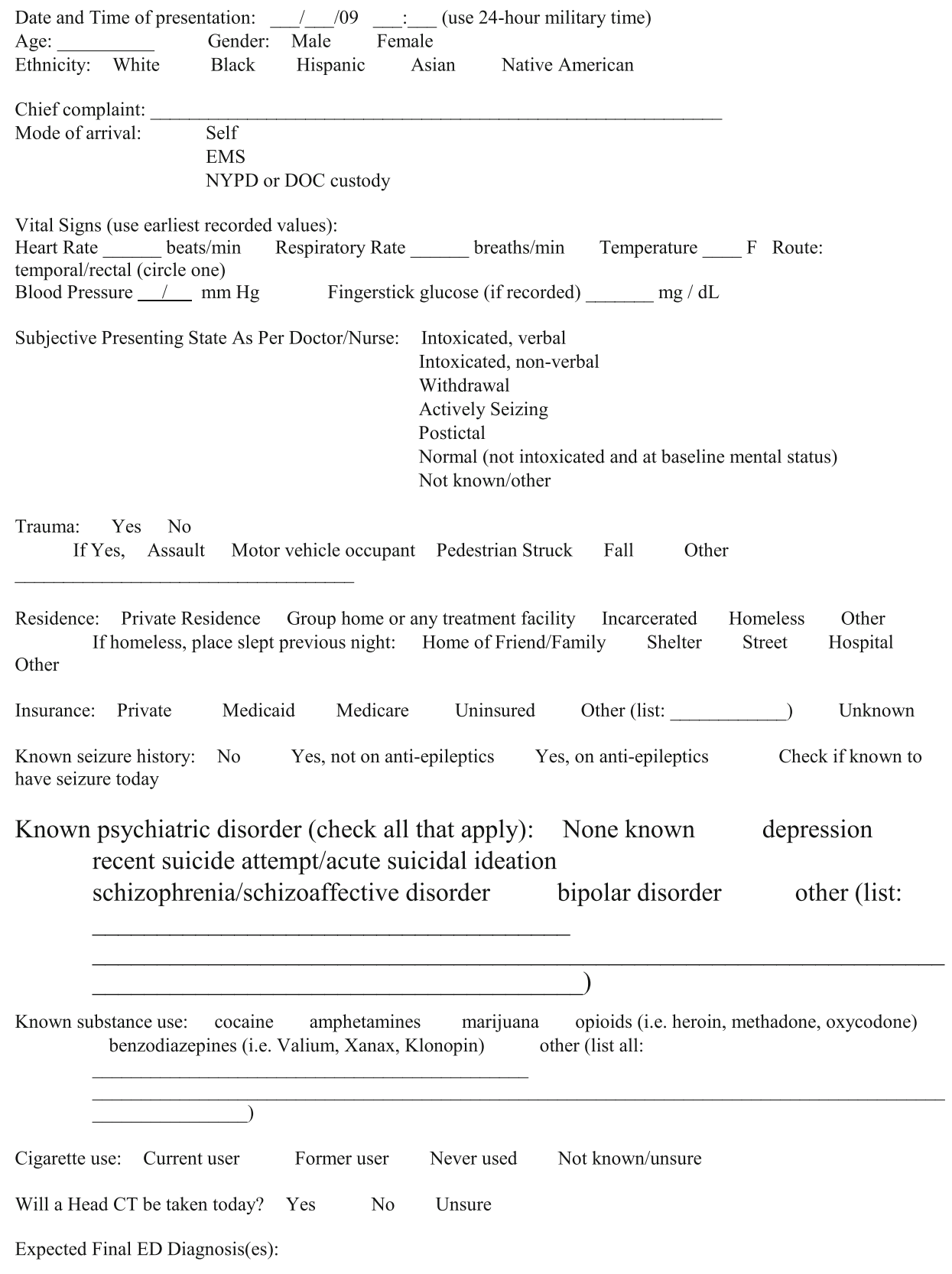

Expected Disposition:

Home

Admission

Medical

Regular bed

Observation bed

ICU bed

Detox (withdrawal management)

Psychiatric

Neurology

Other (specify: 


\section{References}

1. Levine H (1984) The alcohol problem in America: from temperance to alcoholism. Br J Addict 79:109-119

2. Becker B, Woolard R, Nirenberg TD et al (1995) Alcohol use among subcritically injured emergency department patients. Acad Emerg Med 2:784-790

3. Gentilello LM, Donovan DM, Dunn CW et al (1995) Alcohol interventions in trauma centers (current practice and future directions). JAMA 274:1043-1048

4. Cherpitel CJ (1993) Alcohol consumption among emergency room patients: comparison of county/community hospitals and an HMO. J Stud Alcohol 54(4):432-440

5. Whiteman PJ, Hoffman RS, Goldfrank LR (2000) Alcoholism in the emergency department: an epidemiologic study. Acad Emerg Med 7(1):14-20

6. Cherpitel CJ (1988) Drinking patterns and problems associated with injury status in emergency room admissions. Alcohol Clin Exp Res 12:105-110

7. Rockett IR, Putnam SL, Jia H, Smith GS (2003) Assessing substance abuse treatment need: a statewide hospital emergency department study. Ann Emerg Med 41(6):802-813

8. Jolliffe N (1936) The alcoholic admissions to Bellevue Hospital. Science 83:306-309

9. Cherpitel CJ (1994) Injury and the role of alcohol: county-wide emergency room data. Alcohol Clin Exp Res 18(3):679-684

10. Moore RD, Bone LR, Geller G, Mamon JA, Stokes EJ, Levine DM (1989) Prevalence, detection, and treatment of alcoholism in hospitalized patients. JAMA 261:403-407
11. Smothers BA, Yahr HT, Sinclair MD (2003) Prevalence of current DSM-IV alcohol use disorders in short-stay, general hospital admissions, United States, 1994. Arch Intern Med 163:713-719

12. Schneekloth TD, Morse RM, Herrick LM, Suman VJ, Offord KP, Davis LJ (2001) Point prevalence of alcoholism in hospitalized patients: continuing challenges of detection, assessment, and diagnosis. Mayo Clin Proc 76:460-466

13. Smothers BA, Yahr HT, Ruhl CE (2004) Detection of alcohol use disorders in general hospital admissions in the United States. Arch Intern Med 164(7):749-756

14. Priest RG (1976) The homeless person and the psychiatric services: an Edinburgh survey. Br J Psychiatry 128:128-136

15. Kroll J, Carey K, Hagedorn D, Dog PF, Benavides E (1986) A survey of homeless adults in urban emergency shelters. Hosp Community Psychiatry 37(3):283-286

16. Welte JW, Barnes GM (1992) Drinking among homeless and marginally housed adults in New York State. J Stud Alcohol 53 (4):303-315

17. Borges G, Cherpitel CJ, Medina-Mora ME et al (1998) Alcohol consumption in emergency room patients and the general population: a population-based study. Alcohol Clin Exp Res 22:1986-1991

18. Li G, Keyl PM, Rothman R, Chanmugam A, Kelen GD (1998) Epidemiology of alcohol-related emergency department visits. Acad Emerg Med 5(8):788-795

19. Armstrong GL, Conn LA, Pinner RW (1999) Trends in infectious disease mortality in the United States during the 20th century. JAMA 281:61-66

20. Loomis D, Bena J, Bailer A (2003) Diversity of trends in occupational injury mortality in the United States, 1980-96. Inj Prev 9 (1):9-14 